\title{
Choices and kernels in bipolar valued digraphs
}

\author{
Raymond Bisdorff \\ Faculty of Law, Economics and Finance, University of Luxembourg, Avenue de la \\ Faïencerie 162a, L-1511 LUXEMBOURG. \\ Marc Pirlot \\ Faculté Polytechnique de Mons, 9, rue de Houdain, B-7000 Mons, Belgium. \\ Marc Roubens \\ Faculté Polytechnique de Mons, 9, rue de Houdain, B-7000 Mons, Belgium.
}

\begin{abstract}
We explore the extension of the notion of kernel (independent, dominant or absorbent, non-empty subset) of a digraph to valued graphs (or valued relations). We define various natural extensions and show the relationship between them. This work has potential interest for applications in choice decision problems.
\end{abstract}

Keywords: Decision Making, Outranking methods, Choice, Kernel, Digraphs, Valued relations, Fuzzy sets.

\section{Introduction}

Dominant kernels have been initially proposed as solutions of games by von Neumann and Morgenstern (1944). Roy (1968) has suggested to use them as a set of potentially good candidates among which to choose when facing a decision problem. In the latter context, the preference relation on the set of alternatives is usually built using some sort of a majority rule with vetoes (Roy and Bouyssou, 1993), which results in a relation that is not necessarily transitive or complete and may have cycles.

Email addresses: raymond.bisdorff@uni.lu (Raymond Bisdorff), marc.pirlot@fpms.ac.be (Marc Pirlot), roubens@belgacom.net (Marc Roubens). 
In some versions of this process, the preference (outranking) relation is a valued (or fuzzy) one. The value attached to an arc $(a, b)$ may express for instance the degree with which $a$ is preferred to $b$, a credibility index assigned to the preference of $a$ over $b$, etc. In the perspective of generalizing to valued relations the methods used for choosing among a finite set of alternatives on the basis of crisp $(\{0,1\}$-valued) relations, one can think of a generalization of the notion of kernel of valued graphs or relations.

As is usual when studying valued (fuzzy) counterparts of notions that have first been introduced for ordinary (crisp) sets, there are several ways of generalizing the notion of kernel. The main goal of this paper is to explore the relationship between various natural definitions of kernels for valued graphs and see also how they relate to kernels of crisp graphs. Our analysis is restricted to finite graphs.

In section 2, we recall the definitions of kernels in the crisp case, relating mainly the set-theoretic definition (in terms of independent, dominant or absorbent, non-empty subset) and the algebraic one by means of the so-called kernel equation system. Note that we consider two sorts of kernels, the dominant and the absorbent one, that are just dual of one another in the sense that the absorbent kernels of a graph are the dominant kernels of the graph obtained by reversing the orientation of the arcs of the original one.

Section 3 introduces a valuation of the arcs by degrees belonging to an ordered set $L$; this set is equipped with a negation (an antitone one-to-one operation). If $L$ contains an odd number of elements, there is an element that is equal to its negation; this plays the role of an undetermined level "0" that may be used for instance for coding lack of information, like a missing arc in the graph. One distinguishes the levels of $L$ that are above " 0 " (positive) and those below " 0 " (negative). We then assume a graph with arcs assigned a value in $L$. If $L$ has a " 0 ", we may suppose, without loss of generality, that the graph is complete, assigning the value 0 to the missing arcs. This graph is also a valued relation and we use indifferently both terminologies.

The first idea for generalizing kernels, is to cut the relation above 0, yielding a crisp relation or graph, and to make profit of the set-theoretic definition of the kernels of the obtained crisp graph. We call such kernels L-independent, dominant (or absorbent) choices, reserving the term "kernel" for the solutions of the kernel equation system. A variety of "degrees of qualification" are defined and associated to these subsets; they may be interpreted as characterizing their quality as a set of good alternatives (for dominant subsets) or as a set of bad alternatives (for absorbent subsets). We show that $L$-independent, dominant (resp. absorbent) choices can be defined alternatively by means of their degree of qualification as a set of good (resp. bad) alternatives. 
The generalization of the kernel equation system yields solutions, i.e. kernels, that are valued (fuzzy) sets. We identify a subset of solutions, the maximally sharp ones, and we show in section 4 that they correspond to the set of $L$ independent dominant (or absorbent) choices that were initially defined. The proof - using fixpoint argument - is constructive, enabling to find the $L$-valued kernels starting from the independent dominant (or absorbent) choices. The degree of sharpness of the former is shown to be equal to the degree of goodness (or badness) of the latter. From a theoretical point of view, this is rather satisfactory since the various generalizations of the kernel lead to essentially the same notion, though in a non-trivial way, as the reader will see. One should add that there are perspectives, using $L$-valued kernels, to contribute to methods for choosing among a set of alternatives, but such developments are left for another publication.

\section{Kernels of crisp binary relations}

\subsection{Independent dominant and absorbent choices}

In this section, we consider a finite set $X=\{a, b, c, d, \ldots\}$ of alternatives endowed with a binary relation $R$. This relation is interpreted as a preference relation; we do not assume any particular property, such as transitivity, of $R$; the preference may thus result from the aggregation of multiple attribute information through some kind of a majority rule or according with some form of a concordance-discordance principle like they are implemented for instance in the ElECTRE methods (Roy, 1968; Roy and Bouyssou, 1993).

We denote by $a R b$ the fact that the pair $(a, b)$ belongs to the relation $R$; we phrase $a R b$ as " $a$ is preferred to $b$ " (this not excluding that $b$ may also be preferred to $a$; $R$ is not supposed to be an asymmetric relation).

The set $X$ endowed with the relation $R$ can also be viewed as a directed graph (digraph, for short) $G=(X, R)$ where $X$ represents the set of vertices and $(a, b)$ is an arc of the graph if and only if $(a, b) \in R$.

It has been suggested in the ElECTRE I method (Roy, 1969) to use independent dominant subsets of $\mathrm{X}$ as potential good choice if the relation $R$ is acyclic.

Definition 1 (Independent dominant and absorbent choices).

A choice set in the digraph $G=(X, R)$ is a non empty subset $Y$ of $X$.

An independent choice or independent subset in $G$ is a choice set $K \subseteq X$ such 
that $\forall a \neq b \in K,(a, b) \notin R$.

A dominant choice or dominant subset in $G$ is a choice set $K \subset X$ such that $\forall a \notin K, \exists b \in K,(b, a) \in R$.

An absorbent choice or absorbent subset in $G$ is a choice set $K \subset X$ such that $\forall a \notin K, \exists b \in K,(a, b) \in R$.

Example 1. The graph $G_{1}=\left(X_{1}, R_{1}\right)$, where $X_{1}=\{a, b, c, d\}$ and $R_{1}=$ $\{(a, b),(a, c),(a, d),(b, c),(b, d)\}$ is illustrated in figure 1 . There is a unique independent dominant choice ("good choice") that is the singleton $\{a\}$; the unique independent absorbent choice ("bad choice") is $\{c, d\}$.

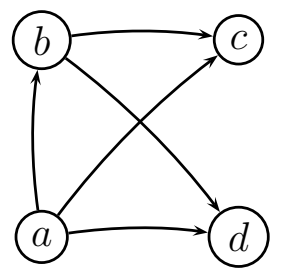

Figure 1. Graph of the preference relation in example 1

Example 2. The graph $G_{2}=\left(X_{2}, R_{2}\right)$, where $X_{2}=\{a, b, c, d\}$ and $R_{2}=$ $\{(a, b),(b, c),(c, d),(d, a)\}$ is illustrated in figure 2. In this example, $\{a, c\}$ and $\{b, d\}$ are two independent choices that are both dominant and absorbent.

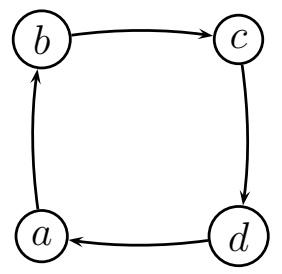

Figure 2. Graph of the preference relation in example 2

If a relation $R$ is acyclic, then it has a unique dominant (resp. absorbent) choice. If, in addition, the relation is transitive, it is a partial order and the set of alternatives that have no predecessor (resp. successor) in the graph forms an independent dominant (resp. absorbent) choice. The above existence and uniqueness property is a motivation for choosing among the alternatives that belong to the independent dominant choice. In case one aims at eliminating "bad" alternatives, a symmetric argument would lead to choosing them in the independent absorbent choice set, again for acyclic digraphs. We loose such an appealing interpretation in case $R$ is not acyclic, as we can see in example 2 above. 


\subsection{Dominant and absorbent kernels}

We now introduce an algebraic definition of a independent dominant (resp. absorbent) choice. Each subset $K \subseteq X$ is associated its characteristic (row) vector $Y: X \rightarrow\{0,1\}$ with

$$
Y(a)= \begin{cases}1 & \text { if } a \in K \\ 0 & \text { otherwise }\end{cases}
$$

for all $a \in X$.

Definition 2 (Dominant and absorbent kernels).

A dominant kernel (Bisdorff and Roubens, 1996a) is a characteristic vector $Y$ that is a solution of the following system of Boolean equations:

$$
(Y \circ R)(a)=\neg Y(a), \quad \text { for all } a \in X,
$$

where $Y \circ R$ is the $\max \min$ product of the Boolean matrices $Y$ and $R$, i.e.

$$
(Y \circ R)(a)=\max _{b \neq a} \min (Y(b), R(b, a)), \quad \text { for all } a \in X
$$

and $\neg$ is the ordinary negation, i.e. $\neg Y(a)=1-Y(a)$. The system of equations (2.2) reads more explicitly as:

$$
\max _{b \neq a} \min (Y(b), R(b, a))=1-Y(a), \quad \text { for all } a \in X .
$$

We call absorbent kernel a characteristic vector $Y$, solution of the system of Boolean equations: for all $a \in X$,

$$
\left(R \circ Y^{t}\right)(a)=\max _{b \neq a} \min (R(a, b), Y(b))=\neg Y(a)=1-Y(a),
$$

where $Y^{t}$ denotes the transposed (column) characteristic vector.

We denote $\mathcal{K}^{\operatorname{dom}}(G)\left(\mathcal{K}^{\text {abs }}(G)\right)$ the possibly empty set of dominant (resp. absorbent) kernels in $G$, i.e. the solutions of the system of equations (2.2) (resp. $(2.4))$.

\section{Proposition 1.}

The set of independent dominant (resp. absorbent) choices of $X$ endowed with the relation $R$ ) is the set of dominant (resp. absorbent) kernels of $G=(X, R)$.

Proof. Let $K$ be a non-empty subset of $X$ and let $Y$ be the characteristic vector of $K$. We denote by $Y_{K}$ (resp. $Y_{\bar{K}}$ ), the part of vector $Y$ corresponding to alternatives in $K$ (resp. out of $K$ ). We split similarly the binary matrix 
$R$ representing the relation $R$ into four parts $R_{K K}, R_{K \bar{K}}, R_{\bar{K} K}, R_{\overline{K K}}$; for instance $R_{K K}$ corresponds to the cases $R(a, b)$ in which both $a$ and $b$ belong to $K$; the three other parts are defined similarly. The system of equation (2.2) is rewritten as

$$
\left[\begin{array}{ll}
Y_{K} & Y_{\bar{K}}
\end{array}\right] \circ\left[\begin{array}{ll}
R_{K K} & R_{K \bar{K}} \\
R_{\bar{K} K} & R_{\overline{K K}}
\end{array}\right]=\left[\bar{Y}_{K} \bar{Y}_{\bar{K}}\right] .
$$

One verifies easily that vector $Y$ exactly characterizes a subset $K$ that is independent and dominant if and only if the above system of equations is satisfied. A similar argument applies to independent absorbent choices and absorbent kernels.

In the sequel we shall use indifferently the terms "independent dominant (resp. absorbent) choices" and "dominant (resp. absorbent) kernels".

\subsection{Historical note}

Following the observation that the independent absorbent choice in an acyclic graph corresponds to the kernel of the associated Grundy function, Riguet (1948) introduced the name "noyau" (kernel) for such a choice set. (Absorbent) kernels were in the sequel extensively studied by Berge $(1958,1970)$ in the context of modeling the Nim game. More results on (absorbent) kernels, concerning solutions of different games, have been reported by Schmidt and Ströhlein (1985, 1989). Recently, Bang-Jensen and Gutin (2001) reviewed the link between kernel-solvability and perfect graphs.

Internally stable and dominating choices, i.e.dominant kernels were originally introduced by J. von Neumann and O. Morgenstern under the name "game solution" in the context of game theory (von Neumann and Morgenstern, 1944). B. Roy proposed the same concept in the context of the multicriteria EleCTRE decision aid methods (Roy, 1968, 1985; Roy and Bouyssou, 1993). Ambiguous choices, i.e. both dominant and absorbent choices at the same time, were proposed by Bisdorff (2002a) as potential cluster candidates in the context of multicriteria clustering.

In the general (non-oriented) theory of graphs, kernels appear under the name of independent dominating sets in Haynes et al. (1998). Our oriented dominant (resp. absorbent) version is there identified as an "inside (resp. outside) semidominating set" or sometimes more simply as an "inkernel" (resp. "outkernel").

The absorbent version of the kernel equation system (2.4) was first introduced by Schmidt and Ströhlein $(1985,1989)$ in the context of their thorough exploration of relational algebra. The dominant version (equation system 2.2) was 
introduced by Kitainik (1993) and subsequently used by Bisdorff and Roubens (1996a,b); Bisdorff (1997).

\section{Kernels in valued digraphs}

\subsection{L-valued binary relations}

Let $X$ be a finite set of alternatives and $\tilde{R}$, a valued binary relation on $X$. We assume that the values assigned by $\tilde{R}$ to each pair of alternatives belong to a finite ordered set $L:\left\{c_{0}, c_{1}, \ldots, c_{M}\right\}$ with $c_{0}<c_{1}<\ldots<c_{M}$. The value $\tilde{R}(a, b) \in L$ is often interpreted as the degree of credibility of the assertion "a is preferred to b" (or " $a$ is at least as good as b"). We call such a relation a $L$-valued binary relation (or shortly $L$-vbr).

The set $L$ is endowed with a negation operator $\neg$ that maps $c_{i}$ onto $c_{M-i}$ for all $i=0, \ldots, M$. This operator has a fixpoint $c_{m}$ iff $M$ is equal to $2 m$, with $m$ a positive integer. Bisdorff (2002a) has forcefully argued that one should take $M$ an even number in practice; the median credibility level $c_{m}$ is then interpreted as a logically undetermined value; it may encode a missing arc. In case $M$ is odd the negation has no fixpoint at all and there is thus no logically undetermined value. From now on, for notational simplicity, we map the set $L$ monotonically onto the following sets of integers, endowed with their natural order:

$$
\begin{array}{ll}
-m,-(m-1), \ldots,-1,0,1, \ldots, m-1, m & \text { if } M=2 m \\
-m,-(m-1), \ldots,-1,1, \ldots, m-1, m & \text { if } M=2 m-1 ;
\end{array}
$$

we use or not the value 0 depending on whether $|L|$ is odd or even, respectively; in case $|L|$ is odd (i.e. $M=2 m=|L|-1$ ), the "median credibility level" $c_{m}$ is mapped onto 0. Since this mapping is an order isomorphism, the operators max and min can be seen as acting indifferently on $L$ or its image on the integers; moreover, the negation operator $\neg$ on $L$ corresponds with taking the opposite of an integer in the image set. We henceforth identify $L$ with the sets of integers defined in (3.1), endowed with their natural order and the negation operator, which is just "taking the opposite". We define the subset of positive levels $L^{>0}$ (resp. negative levels $L^{<0}$ ) as being the set of positive integers $\{1,2, \ldots, m\}$ (resp. negative integers $\{-m, \ldots,-1\}$ ); the set of nonnegative levels $L^{\geq 0}$ (resp. nonpositive levels $L^{\leq 0}$ ) is defined as the complement of $L^{<0}$ (resp. $L^{>0}$ ). Of course, there is no difference between $L^{>0}$ and $L^{\geq 0}$ (or between $L^{<0}$ and $L^{\leq 0}$ ) unless $|L|$ is odd (i.e. $M$ is even); in the latter case, the difference consists of the median credibility level " 0 ". In the sequel, for $x \in L$, we shall write $x>0$ (resp. $<0, \geq 0, \leq 0)$ for $x \in L^{>0}\left(\operatorname{resp} . L^{<0}, L^{\geq 0}, L^{\leq 0}\right)$. 
Interpreting the elements of $L$ as truth values or logical levels, as suggested by Bisdorff (2002a), leads to call the elements of $L^{>0}$, "true levels" and those of $L^{<0}$, "false levels". If $R(a, b)>0$ we could say that the proposition " $(a, b) \in R$ " is $L$-true. If, on the contrary, $R(a, b)<0$, the proposition " $(a, b) \in R$ " is $L$-false. If $R(a, b)=0$, i.e. the median level, we say that the proposition " $(a, b) \in R$ " is L-undetermined. The credibility level of a conjunction (resp. a disjunction) of $L$-valued propositions is obtained by using the "min" (resp. the "max") operator defined on $L$.

Two simple special cases of $L$ are the following. If $L$ has only two elements, we get back to the classical Boolean lattice $L_{2}=\left\{c_{0}, c_{1}\right\}=\{-1,1\} ; c_{0}$ or -1 is the symbol for "false", while $c_{1}$ or +1 is the symbol for "true". The three-valued lattice $L_{3}(M=2 m=2)$ corresponds to the case in which -1 represents "false", 0 represents a logically undetermined level and +1 represents "true".

\subsection{Independent dominant or absorbent choices in a L-valued graph}

We denote by $G^{L}=(X, R)$ a complete $L$-valued digraph with vertex set $X$; an arc $(a, b)$ of $G^{L}$ is assigned the value $R(a, b)$. The simplest way of generalizing the notions of independent dominant (resp. absorbent) choice in $G^{L}$ is through using a cut. Define the application $\tau$ mapping the lattice $L$ onto the Boolean lattice $L_{2}$ as follows: the set of levels $L^{>0}$ are all mapped onto +1 ("true") while the levels of $L^{\leq 0}$ are all mapped onto -1 ("false"). This application extends to a transformation of the $L$-valued graph $G^{L}=(X, R)$ into the ordinary graph $\tau G=(X, \tau R) ;(a, b)$ is an arc of the relation $\tau R$ iff $R(a, b)>0$; otherwise, there is no arc $(a, b)$ in the graph $\tau G$.

Definition 3 (Independent dominant or absorbent choices in $G^{L}$ ). The set $K \subseteq X$ is an independent dominant (resp. absorbent) choice in $G^{L}=(X, R)$ if it is an independent dominant (resp. absorbent) choice in $\tau G=(X, \tau R)$.

Note that with this definition an independent dominant (resp. absorbent) choice in $G^{L}=(X, R)$ is an ordinary subset of $X$ and not a "fuzzy" subset of $X$.

Using the values in $L$, we can define various $L$-valued qualification degrees attached to any non-empty subset $K$ of $X$.

Definition 4 (Degrees of qualification of choices). Let $K$ be a non-empty subset of $X$. The degree of independence of the choice $K$ is defined as

$$
\Delta^{\text {ind }}(K)= \begin{cases}m & \text { if }|K|=1 \\ \min _{\substack{b \neq a \\ b \in K}} \min _{a \in K}\{\neg R(a, b)\} & \text { otherwise. }\end{cases}
$$


$K$ is considered to be $L$-independent if $\Delta^{\text {ind }}(K)>0$.

The degree of dominance of a choice $K$ corresponds to

$$
\Delta^{\text {dom }}(K)=\left\{\begin{array}{lr}
m & \text { if } \mathrm{K}=\mathrm{X}, \\
\min _{a \notin K} \max _{b \in K}\{R(b, a)\} & \text { otherwise. }
\end{array}\right.
$$

$K$ is considered to be $L$-dominant if $\Delta^{\operatorname{dom}}(K)>0$.

The degree of absorbence of a choice $K$ is equal to

$$
\Delta^{\mathrm{abs}}(K)=\left\{\begin{array}{lr}
m & \text { if } \mathrm{K}=\mathrm{X}, \\
\min _{a \notin K} \max _{b \in K}\{R(a, b)\} & \text { otherwise. }
\end{array}\right.
$$

$K$ is considered to be $L$-absorbent if $\Delta^{\text {abs }}(K)>0$.

The qualification of $K$ as being an independent and dominant choice corresponds to

$$
Q^{\mathrm{i}-\operatorname{dom}}(K)=\min \left(\Delta^{\mathrm{ind}}(K), \Delta^{\mathrm{dom}}(K)\right) .
$$

The qualification of $K$ as being an independent and absorbent choice, corresponds to

$$
Q^{\mathrm{i}-\mathrm{abs}}(K)=\min \left(\Delta^{\mathrm{ind}}(K), \Delta^{\mathrm{abs}}(K)\right)
$$

Definition 5 (Potentially good and bad choices). The set $K \subseteq X$ is a potentially good choice if $Q^{\mathrm{i}-\operatorname{dom}}(K) \in L^{>0}$, i.e. if $K$ is $L$-independent and $L$ dominant. The set $K \subseteq X$ is a potentially bad choice if $Q^{\mathrm{i}-\mathrm{abs}}(K) \in L^{>0}$, i.e. if $K$ is $L$-independent and $L$-absorbent.

We denote by $\mathcal{C}^{\mathrm{p}-\text { good }}\left(G^{L}\right)$ (respectively $\mathcal{C}^{\mathrm{p}-\text { bad }}\left(G^{L}\right)$ ) the (possibly empty) set of potentially good (respectively potentially bad) choices in $G^{L}$. The correspondence between potentially good choices (resp. potentially bad choices) and independent dominant (resp. absorbent) choices of $G^{L}$ (definition 3 ) is not entirely straightforward due to the possible existence of the "undetermined" median level 0 .

Proposition 2 (L. Kitainik, 1993).

Let $G^{L}=(X, R)$ be an $L$-valued digraph and let $\tau G=(X, \tau R)$ be its associated crisp digraph. We have:

$$
\mathcal{C}^{\mathrm{i}-\operatorname{dom}}\left(G^{L}\right) \subseteq \mathcal{K}^{\operatorname{dom}}(\tau G)
$$

and

$$
\mathcal{C}^{\mathrm{i}-\mathrm{abs}}\left(G^{L}\right) \subseteq \mathcal{K}^{\mathrm{abs}}(\tau G)
$$

Proof. Consider $K \neq \emptyset$ such that $\Delta^{\text {ind }}(K) \in L^{>0}$. 


$$
\begin{aligned}
\Delta^{\mathrm{ind}}(K) \in L^{>0} & \Leftrightarrow \min _{\substack{b \neq a \\
b \in K}} \min _{a \in K}(\neg R(a, b))>0 \\
& \Leftrightarrow \forall a \neq b \in K: R(a, b)<0 \\
& \Rightarrow \forall a \neq b \in K:(a, b) \notin \tau R \\
& \Leftrightarrow K \text { is an independent choice in } \tau G=(X, \tau R) .
\end{aligned}
$$

Consider now $K \neq \emptyset$ such that $\Delta^{\text {dom }}(K)>0$.

$$
\begin{aligned}
\Delta^{\operatorname{dom}}(K) \in L^{>0} & \Leftrightarrow \min _{a \notin K} \max _{b \in K} R(b, a)>0 \\
& \Leftrightarrow \forall a \notin K, \exists b \in K: R(b, a)>0 \\
& \Leftrightarrow \forall a \notin K, \exists b \in K:(b, a) \in \tau R \\
& \Leftrightarrow K \text { is a dominant set in } \tau G .
\end{aligned}
$$

Using the same arguments,

$$
\Delta^{\mathrm{abs}}(K)>0 \Leftrightarrow K \text { is an absorbent set in } \tau G \text {. }
$$

The proposition is an immediate consequence of the previous results.

From the first part of the previous proof (independence), it clearly appears that the sets $\mathcal{C}^{\text {p-good }}\left(G^{L}\right)$ and $\mathcal{K}^{\operatorname{dom}}(\tau G)$ may be different only if $R(a, b)$ takes the logically undetermined value 0 ; there may indeed exist sets $K \subseteq X$ that are independent dominant choices of $\tau G$ but have a qualification $Q^{\mathrm{i}-\mathrm{dom}}(K)$ equal to 0 . A similar remark holds for independent absorbent choices. The following corollary establishes the precise correspondence between potentially good (resp. potentially bad) choices and independent dominant (resp.absorbent) choices in $\tau G$ (which are also the dominant (resp. absorbent) kernels in $\tau G$, as established in proposition 1).

\section{Corollary 1.}

The set of potentially good (resp. potentially bad) choices in $G^{L}=(X, R)$ is the set of independent dominant (resp. absorbent) choices $K$ in $\tau G=(X, \tau R)$ for which $Q^{\text {i-dom }}(K)>0$, i.e. such that $R(a, b) \neq 0$ for all $a, b \in K$. 
Proof.

Consider the first part of the proof of proposition 2. We have

$$
\Delta^{\text {ind }}(K) \in L^{>m} \Leftrightarrow K \text { is an independent set in } \tau G
$$

iff $R(a, b) \neq 0$ for all $a, b \in K$. The rest of the proof follows that of proposition 2 .

Example 3. The following example (see figure 3) has been suggested by Bernard Roy (Lausanne, 1995; see Bisdorff $(2000)$ ). Let $G^{L}$ be such that $X=$ $\{a, b, c\}, L=\{-5, \ldots, 0, \ldots, 5\}$ and $R:\{R(a, b)=-5, R(b, a)=R(c, a)=$ $R(c, b)=1, R(b, c)=R(a, c)=5\}$.

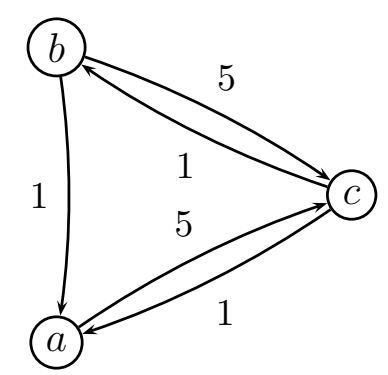

Figure 3. Graph of the preference relation in example 3

Table 1

Degrees of qualification of all choices in example 3

\begin{tabular}{lrrrrr} 
choice & $\Delta^{\text {ind }}$ & $\Delta^{\text {dom }}$ & $\Delta^{\text {abs }}$ & $Q^{\text {i-dom }}$ & $Q^{\text {i-abs }}$ \\
\hline$\{a\}$ & 5 & -5 & 1 & -5 & 1 \\
$\{b\}$ & 5 & 1 & -5 & 1 & -5 \\
$\{c\}$ & 5 & 1 & 5 & 1 & 5 \\
$\{a, b\}$ & -1 & 5 & 1 & -1 & -1 \\
$\{a, c\}$ & -5 & 1 & 5 & -5 & -5 \\
$\{b, c\}$ & -5 & 1 & 5 & -5 & -5 \\
$\{a, b, c\}$ & -5 & 5 & 5 & -5 & -5
\end{tabular}

Table 3 shows the degrees of qualification for all choices in $G^{L}$. All singletons are $L$-independent. All choices, except $\{a\}$, are $L$-dominant and all are $L$-absorbent, except $\{b\}$. Therefore, the singletons $\{b\}$ and $\{c\}$ give potentially good single choices whereas $\{a\}$ and $\{c\}$ give potentially bad single choices. Choices consisting of pairs, as well as the choice consisting of $X$, are neither potentially good nor potentially bad, as they all lack the required $L$-independence condition. Although the relation $R$ often takes the undetermined value $0, \mathcal{C}^{\mathrm{p}-\operatorname{good}}\left(G^{L}\right)=\mathcal{K}^{\operatorname{dom}}(\tau G)$ and $\mathcal{C}^{\mathrm{p}-\text { bad }}\left(G^{L}\right)=\mathcal{K}^{\mathrm{abs}}(\tau G)$. 
One may argue that the indices $Q^{\mathrm{i}-\mathrm{dom}}$ and $Q^{\mathrm{i}-\mathrm{abs}}$ can be used to select a "good choice" among several possible ones; for instance, in example 3, one sees from table 3 that $\{b\}$ is a more convincing potentially good choice than $\{c\}$ since $\{c\}$ is also a potentially bad choice. We refer the reader to Bisdorff and Roubens (2004) for further developments of this idea.

Proposition 2 suggests a way of computing the "good choices" (and the "bad" ones) of $L$-valued graphs: compute the independent dominant choices of $\tau G$; then verify, by computing their degree of independence $\Delta^{\text {ind }}$ and checking whether $\Delta^{\text {ind }}>0$, that they are potentially good choices.

\section{$3.3 \quad$ L-valued dominant and absorbent kernels}

We now address the generalization of the kernel equation systems (2.2) and (2.4). Choices and kernels of $L$-valued relations can be defined as fuzzy sets; the degree of membership of an element $a$ of $X$ to such a set belongs to the set $L$.

A characteristic vector $\tilde{Y}$ of a fuzzy subset of $X$ is an application from $X$ onto $L$, i.e. a row vector $[\tilde{Y}(x), x \in X]$. If $\tilde{Y}$ is to be interpreted as a choice and depending on the context, $\tilde{Y}(a)$, for any $a \in X$, may be interpreted as the degree of truth or the credibility of the assertion "element a belongs to the choice $\tilde{Y}$ ". Values of $\tilde{Y}(a)$ above 0 are interpreted as meaning that $a$ tends more to belong to $\tilde{Y}$ than not; the converse interpretation holds when $\tilde{Y}(a)$ is below $0 ; \tilde{Y}(a)=0$ represents an undetermined situation.

It is rather straightforward to extend the kernel equation systems (2.2) and (2.4) to the case in which $R$ is $L$-valued and we accept solutions that are $L$-valued. These equations become: for all $a \in X$ :

$$
\begin{aligned}
(\tilde{Y} \circ R)(a) & =\max _{b \neq a}[\min (\tilde{Y}(b), R(b, a))]=\neg \tilde{Y}(a)=-\tilde{Y}(a), \\
\left(R \circ \tilde{Y}^{t}\right)(a) & =\max _{b \neq a}[\min (R(a, b), \tilde{Y}(b))]=\neg \tilde{Y}(a)=-\tilde{Y}(a) .
\end{aligned}
$$

Applying the negation operator to the above equations yields the following formulation that will be used in the sequel:

$$
\begin{array}{ll}
\tilde{Y}(a)=\min _{b \neq a}[\max (-\tilde{Y}(b),-R(b, a))], & \forall a \in X \\
\tilde{Y}(a)=\min _{b \neq a}[\max (-R(a, b),-\tilde{Y}(b))], & \forall a \in X .
\end{array}
$$


We denote by $\mathcal{Y}^{\text {dom }}\left(G^{L}\right)$ (resp. $\mathcal{Y}^{\text {abs }}\left(G^{L}\right)$ ) the set of solutions of equation system 3.7 (resp. 3.8).

Example 4. Consider $G^{L}=(X, R)$ with $X=\{a, b, c\}, L=\{-5, \ldots, 0, \ldots, 5\}$ $(M=2 m=10)$ and $R=\{R(a, b)=-3\}, R(a, c)=4, R(b, a)=1, R(b, c)=$ $5, R(c, a)=2, R(c, b)=3\}$ (see figure 4 ).

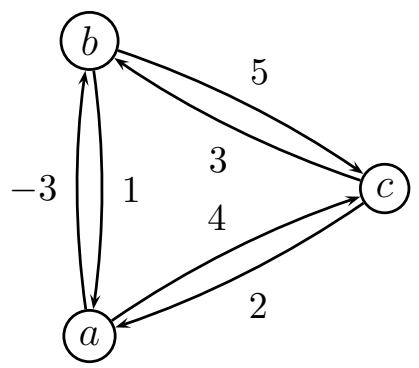

Figure 4. Graph of the preference relation in example 4

The corresponding dominant kernel equation system is:

$$
[\tilde{Y}(a) \tilde{Y}(b) \tilde{Y}(c)] \circ\left[\begin{array}{ccc}
- & -3 & 4 \\
1 & - & 5 \\
2 & 3 & -
\end{array}\right]=[-\tilde{Y}(a)-\tilde{Y}(b)-\tilde{Y}(c)]
$$

Its solutions are shown in table 2 . Solution $\tilde{Y}_{0}$ is completely $L$-undetermined; Table 2

Solutions of Equation System (3.11). The starred solutions are maximally sharp.

\begin{tabular}{rrrr} 
solution & $\tilde{Y}(a)$ & $\tilde{Y}(b)$ & $\tilde{Y}(c)$ \\
\hline$\tilde{Y}_{0}$ & 0 & 0 & 0 \\
\hline$\tilde{Y}_{1}$ & -1 & 1 & -1 \\
$\tilde{Y}_{2}$ & -1 & 2 & -2 \\
$\tilde{Y}_{3}^{*}$ & -1 & 3 & -3 \\
\hline$\tilde{Y}_{4}$ & -1 & -1 & 1 \\
$\tilde{Y}_{5}^{*}$ & -2 & -2 & 2
\end{tabular}

it doesn't characterize any choice at all and we may ignore it. If we apply the operator $\tau$ to the fuzzy characteristic vectors, solutions $\tilde{Y}_{1}, \tilde{Y}_{2}$ and $\tilde{Y}_{3}^{*}$ yield the same crisp choice $\{b\}$, whereas solutions $\tilde{Y}_{4}$ and $\tilde{Y}_{5}^{*}$ yield the same crisp choice $\{c\}$. Note that both subsets of solutions are organized as chains of more and more logically determined solutions, i.e. coming increasingly close to the values -5 and +5 that represent the maximal degree of falseness and truth, respectively. In each group of solutions, we shall focus on the maximal solutions, $\tilde{Y}_{3}^{*}$ and $\tilde{Y}_{5}^{*}$, with respect to this determinateness. 
We formalize the remarks made about the example into precise definitions. We call an $L$-function any $L$-valued function defined on $X$. We mainly consider $L$-functions $\tilde{Y}$ such that $\tilde{Y}(a) \neq 0$, for all $a \in X$; the set of such $L$-functions is denoted by $\mathcal{L}_{0}$.

Definition 6 (Sharpness). Let $\tilde{Y}, \tilde{Z}$ be two functions in $\mathcal{L}_{0}$. We say that $\tilde{Y}$ is at least as sharp as $\tilde{Z}$, which we denote by $\tilde{Z} \preccurlyeq \tilde{Y}$, iff, for all $a \in X$, either $\tilde{Y}(a) \leq \tilde{Z}(a)<0$ or $0<\tilde{Z}(a) \leq \tilde{Y}(a)$. The asymmetric part $\prec$ of $\preccurlyeq$ is defined as follows: $a \prec b$ iff $a \preccurlyeq b$ and not $b \preccurlyeq a$.

The "sharpness" relation $\prec$ is a partial order (asymmetric and transitive relation) on the set of $L$-functions in $\mathcal{L}_{0}$.

To each function $\tilde{Y}$ in $\mathcal{L}$, we associate a crisp subset of X, which we denote by $K_{\tilde{Y}}$ and define by:

$$
K_{\tilde{Y}}=\tau \tilde{Y}=\{a \in X \text { such that } \tilde{Y}(a)>0\} .
$$

We call $K_{\tilde{Y}}$ the strict median cut choice associated with $K_{\tilde{Y}}$.

For any $L$-function $\tilde{Y} \in \mathcal{L}_{0}$, the degree of sharpness $Q^{\text {sharp }}(\tilde{Y})$ of $\tilde{Y}$ is defined as:

$$
Q^{\operatorname{sharp}}(\tilde{Y})=\min \left(\min _{a \in K_{\tilde{Y}}} \tilde{Y}(a), \min _{a \notin K_{\tilde{Y}}}-\tilde{Y}(a)\right) .
$$

This index interprets as a degree of sharpness of $\tilde{Y}$ since we have

$$
\tilde{Y} \preccurlyeq \tilde{Z} \quad \Rightarrow \quad Q^{\operatorname{sharp}}(\tilde{Y}) \leq Q^{\operatorname{sharp}}(\tilde{Z}) .
$$

Of course, we may have $Q^{\operatorname{sharp}}(\tilde{Y}) \leq Q^{\operatorname{sharp}}(\tilde{Z})$ and neither $\tilde{Y} \preccurlyeq \tilde{Z}$ nor $\tilde{Z} \preccurlyeq \tilde{Y}$.

Definition 7 ( $L$-valued dominant and absorbent kernels).

We call $L$-dominant (resp. $L$-absorbent) kernel a $L$ - valued characteristic vector $\tilde{Y}$ that satisfies the following three conditions:

- $\tilde{Y}$ is a solution of equation system (3.7) (resp. (3.8));

- it is maximal with respect to the sharpness relation $\preccurlyeq$ in the set $\mathcal{Y}^{\operatorname{dom}}\left(G^{L}\right)$ (resp. $\mathcal{Y}^{\text {abs }}\left(G^{L}\right)$ ) of admissible solutions of (3.7) (resp. (3.8)), i.e. there is no other solution $\tilde{Z}$ such that $\tilde{Y} \prec \tilde{Z}$;

- it belongs to $\mathcal{L}_{0}$, i.e. $\tilde{Y}(a) \neq 0$, for all $a \in X$

We denote by $\mathcal{F}^{\operatorname{dom}}\left(G^{L}\right)$ (resp. $\mathcal{F}^{\text {abs }}\left(G^{L}\right)$ ) the (possibly empty) set of $L$ dominant ( $L$-absorbent) kernels of $G^{L}$.

Revisiting example 4, we have $\tilde{Y}_{3}^{*}$ and $\tilde{Y}_{5}^{*}$ (see Table 2) as $L$-dominant kernels of $G^{L}$; defuzzyfying $\tilde{Y}_{3}^{*}$ using the operator $\tau$ yields $\tau \tilde{Y}_{3}^{*}=\{b\}$. In a similar way, we get $\tau \tilde{Y}_{5}^{*}=\{c\}$. Those sets are the two dominant kernels of $\tau G$. A similar computation, using the corresponding absorbent kernel equation 
systems yields the solutions $[Y(a)=2, Y(b)=-1, Y(c)=-2]$ and $[Y(a)=$ $-4, Y(b)=-4, Y(c)=4]$ as the $L$-absorbent kernels in $G^{L}$.

In example 3 , the digraph $G^{L}$ admits the set $\mathcal{F}^{\text {i-dom }}\left(G^{L}\right)=\left\{\tilde{Y}_{1}^{*}, \tilde{Y}_{2}^{*}\right\}$ of $L$ dominant kernels and the set $\mathcal{F}^{\text {bad }}\left(G^{L}\right)=\left\{\tilde{Y}_{3}^{*}, \tilde{Y}_{4}^{*}\right\}$ of $L$-absorbent kernels (see table 3 ). Applying the operator $\tau$ to these $L$-valued kernels yields the choice sets that were already obtained for example 3 in section 3.2.

Table 3

$L$-valued kernels in the digraph of Example 3

\begin{tabular}{rrrr} 
kernel & $\tilde{Y}(a)$ & $\tilde{Y}(b)$ & $\tilde{Y}(c)$ \\
\hline$L$-dominant & & & \\
$\tilde{Y}_{1}^{*}$ & -1 & 5 & -5 \\
$\tilde{Y}_{2}^{*}$ & -1 & -1 & 1 \\
\hline$L$-absorbent & & & \\
$\tilde{Y}_{3}^{*}$ & 1 & -1 & -1 \\
$\tilde{Y}_{4}^{*}$ & -5 & -5 & 5
\end{tabular}

From the latter example, it appears that $L$-valued kernels and potentially good or potentially bad choices of a valued graph are strongly related. In the next section, we show the formal correspondence between those notions.

\section{Relating $L$-valued kernels and potentially good and bad choices}

In this section, we shall only deal with the "dominant case"; our results extend mutatis mutandis to the "absorbent case". It is easy to establish a link between a subset of solutions of the dominant kernel equation system (3.7) and a subset of dominant kernels of $\tau G$ that we define below.

Definition 8 (Determined solutions of the dominant kernel equation system). A solution $\tilde{Y}$ of the dominant kernel equation system (3.7) is said determined if it belongs to $\mathcal{L}_{0}$ i.e. $\tilde{Y}(a) \neq 0$, for all $a \in X$. We denote by $\mathcal{Y}_{0}^{\text {dom }}\left(G^{L}\right)$ the set $\mathcal{Y}^{\operatorname{dom}}\left(G^{L}\right) \cap \mathcal{L}_{0}$ of determined solutions of (3.7).

Definition 9 ( $R$-determined subsets). A subset $K$ of $X$ in the valued graph $G^{L}=(X, R)$ is $R$-determined if $R(a, b) \neq 0$ for all $a, b \in K$.

Lemma 1. If $\tilde{Y}$ belongs to $\mathcal{L}_{0}$ and is a solution of the dominant kernel equation system (3.7), then $\tau \tilde{Y}=K_{\tilde{Y}}$ is a $R$-determined dominant kernel of $\tau G$. 
Proof. Using the fact that $\tilde{Y}$ is a solution of the dominant kernel equation system, we apply $\tau$ to both sides of (3.7), yielding

$$
-\tau \tilde{Y}(a)=\max _{b \neq a}[\min (\tau \tilde{Y}(b), \tau R(b, a))]
$$

which is exactly (2.2) with $Y=\tau \tilde{Y}$. It remains to be proven that $K_{\tilde{Y}}$ is $R$-determined. Assume that there are $a, x \in K_{\tilde{Y}}$ with $R(a, x)=0$. Since $\tilde{Y}$ belongs to $\mathcal{L}_{0}$ by hypothesis, $\tilde{Y}(a), \tilde{Y}(x)>0$. Using the "negative form" (3.9) of the kernel equation system, we have for that particular $a \in K_{\tilde{Y}}$ :

$$
0<\tilde{Y}(a) \leq \max (-\tilde{Y}(x),-R(x, a))=0,
$$

a contradiction.

\subsection{A useful transformation}

The elements of $\mathcal{Y}_{0}^{\text {dom }}\left(G^{L}\right)$ i.e. the determined solutions of the dominant kernel equation system can be viewed as the fixpoints of a transformation $T$ that operates on $\mathcal{L}_{0}$.

Definition 10 (The transformation $T$ ). The transformation $T$ maps a function $\tilde{U} \in \mathcal{L}_{0}$, onto $T \tilde{U}$ defined by:

$$
T \tilde{U}(a)=\min _{x \neq a} \max (-\tilde{U}(x),-R(x, a)), \quad \forall a \in X .
$$

The fixpoints of $T$ in $\mathcal{L}_{0}$ are the solutions of (3.9) or, equivalently, of (3.7), since

$$
T \tilde{U}=\tilde{U} \quad \Leftrightarrow \quad \tilde{U}(a)=\min _{x \neq a} \max (-\tilde{U}(x),-R(x, a)), \quad \forall a \in X .
$$

Lemma 2. If $K$ is a $R$-determined dominant kernel of $\tau G$ and $\tilde{U} \in \mathcal{L}_{0}$ is such that $K_{\tilde{U}}=K$, then $T \tilde{U} \in \mathcal{L}_{0}$ and $K_{T \tilde{U}}=K$. Moreover, for all $a \in K_{\tilde{U}}$, we may compute $\tilde{U}($ a) as follows:

$$
T \tilde{U}(a)=\left(\min _{x \neq a ; x \in K_{\tilde{U}}}-R(x, a)\right) \wedge\left(\min _{x \notin K_{\tilde{U}}} \max (-\tilde{U}(x),-R(x, a))\right)
$$

and for all $a \notin K_{\tilde{U}}$, we have:

$$
T \tilde{U}(a)=\min _{x \in K_{\tilde{U}}} \max (-\tilde{U}(x),-R(x, a))
$$

Proof. If $a \in K, T \tilde{U}(a)>0$ because 
- for $x \in K, R(x, a)<0$ and thus, for $x \in K, \max (\neg \tilde{U}(x),-R(x, a))=$ $-R(x, a)$ (this also proves that(4.1) is equivalent to (3.9), for $a \in K$ );

- for all $x \notin K, \max (-\tilde{U}(x),-R(x, a)) \geq-\tilde{U}(x)>0$.

If $a \notin K, T \tilde{U}(a)<0$ because there is $x \in K$ such that $R(x, a)>0$; for such an $x, \max (-\tilde{U}(x),-R(x, a))<0$ and hence $\tilde{U}(a)<0$. Moreover, for all $x$ not in $K, \max (-\tilde{U}(x),-R(x, a))>0$, hence (4.2) is valid.

Corollary 2. Let $\tilde{Y} \in \mathcal{L}_{0}$. The function $\tilde{Y}$ is a solution of equation system (3.9) (or equivalently of (3.7)), iff $\tilde{Y}$ satisfies

$$
\tilde{Y}(a)=\left(\min _{x \neq a ; x \in K_{\tilde{Y}}}-R(x, a)\right) \wedge\left(\min _{x \notin K_{\tilde{Y}}} \max (-\tilde{Y}(x),-R(x, a))\right),
$$

for all $a \in K_{\tilde{Y}}$, and

$$
\tilde{Y}(a)=\min _{x \in K_{\tilde{Y}}} \max (-\tilde{Y}(x),-R(x, a)),
$$

for all $a \notin K_{\tilde{Y}}$.

Proof.

$[\Rightarrow] \quad$ Lemma 1 indicates that $\tau \tilde{Y}=K_{\tilde{Y}}$ is a $R$-determined dominant kernel of $\tau G$; therefore $\tilde{Y}$ fulfills the hypotheses of lemma 2 yielding the result.

$[\Leftarrow] \quad$ We prove that $K_{\tilde{Y}}$ is a $R$-determined dominant kernel of $\tau G$. In view of (4.3) and the fact that $\tilde{Y}(a)>0$ whenever $a \in K_{\tilde{Y}}$, we deduce that $R(x, a)<0$ for all $a, x \in K_{\tilde{Y}}$. Similarly, for all $a \notin K_{\tilde{Y}}$, we deduce from $\tilde{Y}(a)<0$ and equation (4.4) that there exists at least one $x$ in $K_{\tilde{Y}}$ such that $R(x, a)>0$. Henceforth, $K_{\tilde{Y}}$ is a $R$-determined dominant kernel of $\tau G$. Using the fact that $K_{\tilde{Y}}$ is a kernel of $\tau G$, lemma 2 yields that $T \tilde{Y}$ can be computed using (4.1) and (4.2); by hypothesis, $\tilde{Y}$ satisfies (4.3) and (4.4); therefore $\tilde{Y}$ is a fixpoint of $T$, hence $\tilde{Y}$ belongs to $\mathcal{Y}_{0}^{\operatorname{dom}}\left(G^{L}\right)$.

\subsection{Obtaining a L-dominant kernel from a $R$-determined dominant kernel of $\tau G$}

We use transformation $T$ to obtain a $L$-dominant kernel from a $R$-determined dominant kernel of $\tau G$. The main result we need is the fact that $T$ respects the relation "at least as sharp as" on any subset of $L$-functions $\tilde{Y}$ such that $\tau \tilde{Y}$ is a $R$-determined dominant kernel.

Lemma 3. Let $K$ be a $R$-determined dominant kernel of $\tau G$ and $\tilde{U}, \tilde{V} \in \mathcal{L}_{0}$ be such that $K_{\tilde{U}}=K_{\tilde{V}}=K$ and $\tilde{U} \preccurlyeq \tilde{V}$. Then $T \tilde{U} \preccurlyeq T \tilde{V}$. 
Proof. For any $a \in K$,

$$
T \tilde{V}(a)=\left(\min _{x \neq a ; x \in K_{\tilde{V}}}-R(x, a)\right) \wedge\left(\min _{x \notin K_{\tilde{V}}} \max (-\tilde{V}(x),-R(x, a))\right) .
$$

Since the functions min and max are nondecreasing and for all $x \notin K$, $-\tilde{V}(x) \geq-\tilde{U}(x)$, we get $T \tilde{V}(a) \geq T \tilde{U}(a)$.

For any $a \notin K, T \tilde{V}(a)=\min _{x \in K_{\tilde{V}}} \max (-\tilde{V}(x),-R(x, a))$. Since the functions min and max are nondecreasing and for all $x \in K,-\tilde{V}(x) \leq-\tilde{U}(x)$, we get $T \tilde{V}(a) \leq T \tilde{U}(a)$.

Starting from the characteristic function of a $R$-determined dominant kernel of $\tau G$ and applying iteratively $T$ to this function, one obtains, after a finite number of iterations, an element of $\mathcal{Y}_{0}^{\text {dom }}\left(G^{L}\right)$ that is at least as sharp as any other element $\tilde{Y}$ of $\mathcal{Y}_{0}^{\operatorname{dom}}\left(G^{L}\right)$ such that $K_{\tilde{Y}}=K$.

Proposition 3. Let $K$ be a $R$-determined dominant kernel of $\tau G$ and $\tilde{U} \in \mathcal{L}_{0}$ be defined by $\tilde{U}(a)=m$ if $a \in K$ and $-m$ otherwise. For some finite integer $n, T^{n} \tilde{U}=T^{n+1} \tilde{U}$ and $\tilde{Z}=T^{n} \tilde{U}$ is an element of $\mathcal{Y}_{0}^{\operatorname{dom}}\left(G^{L}\right)$ such that $K_{\tilde{Z}}=K$; we have furthermore $\tilde{Y} \preccurlyeq \tilde{Z}$ for all element $\tilde{Y}$ of $\mathcal{Y}_{0}^{\text {dom }}\left(G^{L}\right)$ with $K_{\tilde{Y}}=K$.

Proof. The function $\tilde{U}$ defined in the proposition belongs to $\mathcal{L}_{0}$. Since, by lemma $2, K_{T \tilde{U}}=K_{\tilde{U}}$, it is obvious from the definition of $\tilde{U}$ that $T \tilde{U} \preccurlyeq \tilde{U}$. Applying lemma 3, we get $T^{i+1} \tilde{U} \preccurlyeq T^{i} \tilde{U}$, for all $i$. Due to the finiteness of $L$ and $X$, there is a finite number $n$ such that $T^{n+1} \tilde{U}$ is not strictly less sharp than $T^{n} \tilde{U}$, which means that $T^{n+1} \tilde{U}=T^{n} \tilde{U}$. For such a $n, \tilde{Z}=T^{n} \tilde{U}$ is an element of $\mathcal{Y}_{0}^{\text {dom }}\left(G^{L}\right)$. It is moreover a maximal element w.r.t. the relation $\preccurlyeq$ in the subset of elements $\tilde{Y}$ of $\mathcal{Y}_{0}^{\operatorname{dom}}\left(G^{L}\right)$ such that $K_{\tilde{Y}}=K$. Let $\tilde{Y}$ be such an element. We have $T \tilde{Y}=\tilde{Y}$ and $\tilde{Y} \preccurlyeq \tilde{U}$. Applying lemma 3, we get $T^{n} \tilde{Y} \preccurlyeq T^{n} \tilde{U}$, which means that $\tilde{Y} \preccurlyeq \tilde{Z}$.

It was not evident at first sight that, for all $R$-determined dominant kernels $K$ of $\tau G$, there are solutions $\tilde{Y}$ of (3.9) with $K_{\tilde{Y}}=K$. It was not evident either that the set of solutions $\tilde{Y}$ of (3.9) with $K_{\tilde{Y}}=K$, when non-empty, admits a unique maximally sharp element. Putting together lemma 1 and proposition 3 , we establish the existence of a bijective correspondence between $R$-determined dominant kernels of $\tau G$ and determined $L$-dominant kernels of $G^{L}$.

Theorem 1. $K$ is a $R$-determined dominant kernel of $\tau G$ iff there is a determined $L$-dominant kernel $\tilde{Y}$ such that $K_{\tilde{Y}}=K$. This $L$-dominant kernel is unique. 


\subsection{Qualification of a dominant kernel}

There is a link between the qualification $Q^{\mathrm{i} \text {-dom }}(K)$ of a dominant kernel $K$ of $\tau G$ and the sharpness qualification of all elements $\tilde{Y}$ of $\mathcal{Y}_{0}^{\text {dom }}\left(G^{L}\right)$ such that $K_{\tilde{Y}}=K$. We first prove that the degree of sharpness of an element of $\mathcal{Y}_{0}^{\text {dom }}\left(G^{L}\right)$ is not larger than the qualification of the corresponding $R$-determined dominant kernel.

Lemma 4. If $K$ is a $R$-determined dominant kernel of $\tau G$, any determined solution $\tilde{Y}$ of the kernel equation system (3.7) is such that the choice $K_{\tilde{Y}}=K$ in $\tau G$ verifies:

$$
Q^{\text {sharp }}(\tilde{Y}) \leq Q^{\text {i-dom }}(K)
$$

Proof. Assume that $Q^{\text {sharp }}(\tilde{Y})>Q^{\mathrm{i} \text {-dom }}(K)$. Let $Q^{\text {sharp }}(\tilde{Y})=r$ and $Q^{\text {i-dom }}(K)=$ $k$, with $r>k>0$. The qualification $Q^{\mathrm{i}-\operatorname{dom}}(K)=k$ is equal either to $-R(b, a)$ for some $a, b \in K$ or to $\max _{x \in K} R(x, y)$, for some $y \notin K$.

In the former case, using (4.3), we get $r \leq \tilde{Y}(a) \leq-R(b, a)=k$, a contradiction.

In the latter case, there is $a \in K$ such that $R(a, y)=\max _{x \in K} R(\underset{\tilde{Y}}{x}, y)=$ $k$. Therefore, for all $x \in K, R(x, y) \leq k$. Using (4.4), we have $\tilde{Y}(y)=$ $\min _{x \in K_{\tilde{Y}}} \max (-\tilde{Y}(x),-R(x, y))$. For all $x \in K,-\tilde{Y}(x) \leq-r \leq-k \leq-R(x, y)^{\dagger \dagger}$; therefore $\tilde{Y}(y) \geq-k$. But, by definition of $Q^{\text {sharp }}(\tilde{Y}), \tilde{Y}(y) \leq-r$ hence $-r \geq \tilde{Y}(y) \geq-k$, a contradiction.

We finally prove that the sharpness of the $L$-dominant kernel $\tilde{Y}$ corresponding to a $R$-determined dominant kernel $K$ is equal to the qualification of $K$.

Theorem 2. For any $R$-determined dominant kernel $K$, the $L$-dominant kernel $\tilde{Z}$ corresponding to $K$ is such that $Q^{\text {sharp }}(\tilde{Z})=Q^{\text {i-dom }}(K)$.

Proof. In view of lemma 4 , it suffices to prove that $Q_{\tilde{W} \text { sharp }}(\tilde{Y}) \geq Q^{\text {i-dom }}(K)$. Let $Q^{\text {i-dom }}(K)=k$, with $k>0$. Define the $L$-function $\tilde{W} \in \mathcal{L}_{0}$ as

$$
\tilde{W}(a)=\left\{\begin{aligned}
k & \text { if } a \in K \\
-k & \text { if } a \notin K .
\end{aligned}\right.
$$

By lemma 2, $T \tilde{W}$ belongs to $\mathcal{L}_{0}$ and $K_{T \tilde{W}}=K_{\tilde{W}}=K$. Using (4.1) and (4.2),

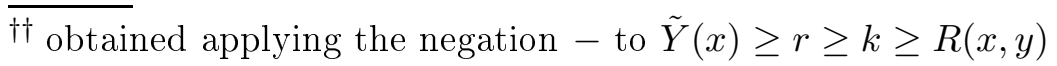


we show that $\tilde{W} \preccurlyeq T \tilde{W}$. Indeed, for $a \in K$,

$$
T \tilde{W}(a)=\left(\min _{x \neq a ; x \in K_{\tilde{W}}}-R(x, a)\right) \wedge\left(\min _{x \notin K_{\tilde{W}}} \max (-\tilde{W}(x),-R(x, a)) \geq k\right),
$$

and, for $a \notin K$,

$$
\begin{aligned}
T \tilde{W}(a) & =\min _{x \in K_{\tilde{W}}} \max (-\tilde{W}(x),-R(x, a)) \\
& =\max \left(-k, \min _{x \in K_{\tilde{W}}}-R(x, a)\right) \\
& \leq-k
\end{aligned}
$$

Remember the definition of $\tilde{U} \in \mathcal{L}_{0}$ that was used in proposition $3: \tilde{U}(a)=m$ if $a \in K$ and $-m$ otherwise. We have $\tilde{W} \preccurlyeq \tilde{U}$ and, using lemma $3, T^{i} \tilde{W} \preccurlyeq T^{i} \tilde{U}$, for all $i=1,2, \ldots$. Since, for some $n, T^{n} \tilde{U}=\tilde{Z}$, the $L$-dominant kernel corresponding to $K$, we have:

$$
\tilde{W} \preccurlyeq T^{n} \tilde{W} \preccurlyeq T^{n} \tilde{U}=\tilde{Z}
$$

and, using (3.14), $Q^{\operatorname{sharp}}(\tilde{W})=k \leq Q^{\operatorname{sharp}}(\tilde{Z})$.

\subsection{Remarks}

The above results prompt the following remarks.

(1) The undetermined value 0 , when such a value appears in the set $L$, plays a special role and has to be carefully dealt with. In lemma 2 for instance, if we either drop the restriction that the dominant kernel $K$ of $\tau G$ is a $R$-determined one or that $\tilde{U}(x) \neq 0$ for all $x \in X$, we can no longer make sure that $T \tilde{U}(a)>0$ for all $a \in K$. This means that the transformed characteristic function $T \tilde{U}$ would not satisfy $K_{T \tilde{U}}=K_{\tilde{U}}$. Since we are interested in determined solutions of the kernel equation system, we did not pay attention to undetermined solutions, and correlatively, we considered only the $R$-determined dominant choices of $\tau G$.

(2) Throughout this paper, we have assumed that both the set $X$ and the set $L$ are finite. Our results extend without difficulty to the case in which $L$ is infinite, while the set $X$ of alternatives remains finite. In such a case, the number of values taken by the relation $R$ is at most $|X| \times(|X|-1)$; let $m$ denote the maximum of $|R(a, b)|$, for $a, b \in X$; the construction of a fixpoint of $T$ as described in proposition 3 remains valid, since $T \tilde{U}$ will only take values also taken by $R$ or their opposite, hence a finite subset of $L$. In case the number of alternatives $X$ is infinite and $L$ is an interval 
of the real numbers (for instance the interval $[-1,1]$ ), the construction of the fixpoint as in proposition 3 still works but the convergence might take an infinite number of steps.

\section{Conclusion}

This paper aims at exploring the relationship between independent dominant (resp. absorbent) choices and solutions of the kernel equation system in the case of valued relations. We have shown that there is a one-to-one correspondence between $L$-dominant (resp. $L$-absorbent) kernels of a valued graph and the ( $R$-determined) independent dominant (resp. absorbent) choices of the strict median cut digraph $\tau G$. Furthermore, starting with an independent dominant (resp. absorbent) choice of $\tau G$, the technique of proof provides a way of building the corresponding $L$-dominant (resp. $L$-absorbent) kernel as the fixpoint of a transformation. This has an important consequence from the algorithmic point of view: finding $L$-valued kernels is not more difficult than finding the kernels of a crisp graph. Indeed, starting with a ( $R$-determined) kernel of $\tau G$ and applying the transformation (definition 10) at most $m|X|$ times yields the fixpoint. Of course, this does not make the determination of the $L$-valued kernels an easy task since we know that finding all kernels of a crisp relation is a computationally hard problem (this was proven by $\mathrm{V}$. Chvátal; see Garey and Johnson (1979), p.204).

Theorem 2 also has interest in its own: the degree of sharpness of a $L$-dominant kernel $\tilde{Y}$ is equal to the degree of potential goodness $Q^{\mathrm{i}-\text { dom }}$ of the associated crisp kernel $K_{\tilde{Y}}$. The comparison of crisp kernels (or equivalently of independent dominant or absorbent choices of $\tau G$ ) done at the end of section 4 about examples 3 and 4 can thus be transposed to $L$-valued kernels.

\section{References}

Bang-Jensen J. and Gutin, G., Digraphs: Theory, algorithms and applications. Springer-Verlag, London, 2001.

Berge, C., Théorie des graphes et ses applications. Dunod, Paris, 1958.

Berge, C., Graphes et hypergraphes. Dunod, Paris, 1970.

Bisdorff, R. and Roubens, M., On defining fuzzy kernels from $L$-valued simple graphs. In: Proceedings Information Processing and Management of Uncertainty, IPMU'96, Granada, July 1996, 593-599.

Bisdorff, R. and Roubens, M., On defining and computing fuzzy kernels from $L$-valued simple graphs. In: Da Ruan et al., eds., Intelligent Systems and Soft 
Computing for Nuclear Science and Industry, FLINS'96 workshop. World Scientific Publishers, Singapoure, 1996, 113-123.

Bisdorff, R., On computing kernels from $L$-valued simple graphs. In: Proceedings 5th European Congress on Intelligent Techniques and Soft Computing EUFIT'97, (Aachen, September 1997),vol. 1, 97-103.

Bisdorff, R., Logical foundation of fuzzy preferential systems with application to the ElECTRE decision aid methods, Computers \& Operations Research, 27, 2000, 673-687.

Bisdorff, R., ELECTRE-like clustering from a pairwise fuzzy proximity index. European Journal of Operational Research, 138/2, 2002, 320-331.

Bisdorff, R. and Roubens, M., Le temps des noyaux .... In: Bair, J. and Henry, V. (ed.) Regards croisés sur les méthodes quantitatives de gestion. Les Éditions de l'Université de Liège, Liège (Belgium), 2004, pp. 41-54.

Garey, M.R. and Johnson, D.S., Computers and intractability, A guide to the theory of NP-Completeness. Freeman and Co., New York, 1979.

Haynes, T.W., Hedetniemi, S.T. and Slater, P.J., Fundamentals of domination in graphs. Marcel Dekker Inc. New-York, 1998.

Kitainik, L., Fuzzy decision procedures with binary relations: towards a unified theory. Kluwer Academic Publ., Boston, 1993.

Riguet, J., Relations binaires, fermetures, correspondances de Galois. Bull. Soc. Math. France, 76, 1948, 114-155.

Roy, B., Classement et choix en présence de points de vue multiples. RIRO 8, $57-75$.

Roy, B., Algèbre moderne et théorie des graphes. Dunod, Paris, 1969.

Roy, B., Méthodologie Multicritère d'aide à la décision. Economica, Paris, 1985.

Roy, B. and Bouyssou, D., Aide multicritère à la décision: Méhodes et cas. Economica, Paris, 1993.

Schmidt, G. and Ströhlein, Th., On kernels of graphs and solutions of games: a synopsis based on relations and fixpoints. SIAM, J. Algebraic Discrete Methods, 6, 1985, 54-65.

Schmidt, G. and Ströhlein, Th., Relationen und Graphen. Springer-Verlag, Berlin, 1989. English version: Relations and graphs, Springer-Verlag, Berlin, 1993.

von Neumann, J. and Morgenstern, O., Theory of games and economic behaviour. Princeton University Press, Princeton 1944. 